\title{
COMPARISON OF TWO MINIMALLY INVASIVE METHODS ON THE LONGEVITY OF GLASS IONOMER CEMENT RESTORATIONS: SHORT-TERM RESULTS OF A PILOT STUDY
}

\author{
Terezinha Jesus Esteves BARATA ${ }^{1}$, Eduardo BRESCIANI ${ }^{2}$, Maria Cecília Ribeiro MATTOS ${ }^{3}$, \\ José Roberto Pereira LAURIS ${ }^{4}$, Dan ERICSON ${ }^{5}$, Maria Fidela de Lima NAVARRO ${ }^{6}$
}

1-DDS, MSc, PhD, Assistant Professor, Department of Operative Dentistry, University of North of Paraná - UNOPAR, PR, Brazil.

2- DDS, MSc, PhD, Assistant Professor, Department of Cariology, Restorative Sciences and Endodontics, School of Dentistry, University of Michigan, MI, USA.

3- DDS, MSC, PhD Student, Department of Operative Dentistry, Endodontics and Dental Materials, Bauru School of Dentistry, University of São Paulo, Bauru, SP, Brazil.

4- PhD, Associate Professor, Department of Pediatric Dentistry, Orthodontics and Public Health, Bauru School of Dentistry, University of São Paulo, Bauru, SP, Brazil.

5- DDS, PhD, Professor, Department of Cariology, Faculty of Odontology, Malmö University, Sweden.

6- DDS, PhD, Professor, Department of Operative Dentistry, Endodontics and Dental Materials, Bauru School of Dentistry, University of São Paulo, Bauru, SP, Brazil.

Corresponding address: Profa. Dra. Maria Fidela Lima Navarro - Faculdade de Odontologia de Bauru, Universidade de São Paulo. Alameda Dr. Octávio Pinheiro Brisolla 9-75, 17012-901, Bauru, SP, Brasil. Phone: 5514 3235-8325. Fax: 55 14 3223 4679. E-mail: mflnavar@usp.br

Received: September 26, 2007 - Modification: November 19, 2007 - Accepted: November 23, 2007

\begin{abstract}
The

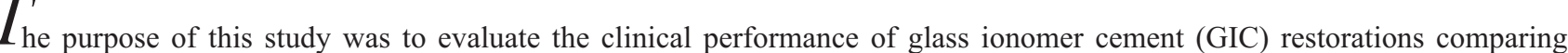
two minimally invasive methods in permanent teeth after 12 months. Fifty pregnant women (second trimester of pregnancy), mean age $22 \pm 5.30$ years, were treated by two previously trained operators. The treatment approaches tested were: chemomechanical method (Carisolv ${ }^{\mathrm{TM}}$; MediTeam) and atraumatic restorative treatment (ART). A split-mouth study design was used in which the two treatments were randomly placed in 50 matched pairs of permanent teeth. The chemomechanical method (CM) was the test group and the ART was the control group. The treatments were performed in Public Health Centers. The tested restorative material was a high-strength GIC (Ketac Molar; 3M/ESPE). The restorations were placed according to the ART guidelines. Two calibrated independent examiners evaluated the restorations in accordance with ART criteria. The interexaminer kappa was 0.97. Data were analyzed using $95 \%$ confidence interval on the binomial distribution and Fisher's exact test at $5 \%$ significance level. In a 12 -month follow-up, $86 \%$ of the restorations were evaluated. In the test group (CM), $100 \%$ $(\mathrm{CI}=93.3-100 \%)$ of the restorations were considered successful. In the control group (ART) 97.6\% (CI=87.4-99.9\%) of the restorations were considered successful and $2.4 \%$ unsuccessful (marginal defect $>0.5 \mathrm{~mm}$ ). There was no statistically significant difference between the 12-mounth success rate for both groups (Fisher's exact test: $\mathrm{P}=0.49$ ) and between the two operators (Fisher's exact test: $\mathrm{P}=1.00$ ). Both minimally invasive methods, chemomechanical method and ART, showed a similar clinical performance after 12 months of follow up.
\end{abstract}

Key words: Clinical trials. Restorations. Glass ionomer cements. Atraumatic Restorative Treatment. Chemomechanical method. Carisolv.

\section{INTRODUCTION}

Current dental restorative concepts are characterized by an increased effort towards a less invasive treatment of caries lesions ${ }^{26}$. Due to the disadvantages of using traditional rotary instruments, such as heat, pressure, dentin desiccation, vibration and pain ${ }^{2,19}$, there has been a considerable interest in developing alternative methods for caries removal ${ }^{3,9}$. This has been possible with better understanding of the etiology and prevention of dental caries, as well as development of adhesive restorative materials ${ }^{26}$. Several approaches for minimally invasive cavity preparation and restorative methods have been developed to preserve as much sound enamel and dentin as possible during treatment of carious lesions ${ }^{2,3,9,26}$. These include the atraumatic restorative treatment (ART) and the chemomechanical method $(\mathrm{CM})^{3,8,9}$.

ART is an approach to the management of dental caries, 
originally developed to provide dental treatment outside the traditional clinical setting ${ }^{9}$. It combines the preventive component with the restorative procedure. ART involves the removal of infected tooth tissues with hand instruments, followed by cavity restoration and sealing of adjacent pits and fissures with glass ionomer cements $(\mathrm{GICs})^{9}$. On the other hand, when caries is excavated with the $\mathrm{CM}$, the active components of the softening agents are mixed and applied to the lesion ${ }^{3}$. The carious tissue is then softened and can be scraped off with hand instruments ${ }^{3}$. This procedure is repeated until all carious tissue has been removed ${ }^{3}$.

Since its introduction, the ART approach has been subjected to research. A large number of these studies have investigated the survival of GIC restorations ${ }^{4,5,10,13,18,25}$. Nevertheless, little is known about the performance of restorations placed on caries-affected dentin excavated with the CM permanent teeth ${ }^{11}$. This is mainly because most studies with CM have been conducted with respect to caries removal, caries removal time, pain and need for anesthesia ${ }^{8,10,12,20}$. There is therefore the need to undertake further studies comparing the use of the ART and CM approaches with respect to longevity of GICs restorations in permanent teeth.

Another aspect to be addressed is the use of minimally invasive cavity preparation in special groups with negative behavior regarding conventional caries excavation, such as pregnant women. In many cases avoidance of dental treatment is justified by popular beliefs that the dental treatment uses rotary instruments and that local anesthetic may be harmful to the embryo ${ }^{1}$. It has also been observed that a significant proportion of the women who were unable to see a dentist or who, once examined, were not treated, received the explanation that such treatment should be avoided during pregnancy ${ }^{21}$. Considering this reality, perhaps the decision to avoid dental care among pregnant women may be modified by minimally invasive operative approaches.

The aim of the present study was to evaluate the clinical performance of GIC restorations comparing two cavity preparation methods in permanent teeth of pregnant women within a 12-month period. The ART was used as a control group and the CM as the test group. The null hypothesis to be tested was that there is no difference in the survival rate of GICs restorations with both cavity preparation methods in the permanent dentition after 12 months.

\section{MATERIALAND METHODS}

\section{Study Design}

The institutional Ethics Committee approved this study (protocol \#7032/2002), according to the guidelines of the Declaration of Helsinki. The patients were informed of the study purposes and were free to decide whether they would participate or not.

The inclusion criteria were: pregnant women (second trimester of pregnancy), presence of two dentinal caries lesions, which had an opening wide enough for the smallest excavator to enter $(\varnothing=0.9 \mathrm{~mm})$. The exclusion criteria were: pulp exposure, history of pain, presence of swelling or fistula and cases judged to be unrestorable according to ART guidelines ${ }^{9}$. Patients with teeth in such conditions were advised to seek care in a Basic Health Center where the adequate treatment was performed. Patients were included in this study only after signing an informed consent form. In case of more than 2 eligible teeth in the same individual, similarity of the size of the cavity and the most posterior position were the criteria used.

For this pilot study, one Public Health Center specialized in the treatment of pregnant women was selected. Two operators and one chair-side assistant performed the treatments. Two blinded evaluators inspected the completeness of caries removal after cavity preparation. Before the start of the study, the operators and the evaluators were trained and calibrated on criteria for complete caries removal. Training and calibration were performed in laboratory during 1 week, and the clinical pilot study was performed in 4 patients during 2 weeks. The intra- and interexaminer reproducibility of complete caries removal status showed very good reproducibility with kappa values of 0.92 and 0.87 , respectively. Prior to being treated, all patients received individually instructions regarding oral health and how to clean their teeth by trained oral health educators.

\section{Randomization}

The pilot study was a clinical randomized controlled trial with split-mouth design, where the two methods were compared in each individual, one immediately after the other, in 50 pairs of permanent teeth. An independent supervisor was responsible for the randomization procedures and the overall logistics of the clinical procedures. After pre-treatment information was retrieved and the patients were found to fulfill the eligibility criteria, including agreement to participate, the caries lesions for each patient were randomized using the following procedure: 1) Sequence of the methods: randomization was carried out by the sealed envelope technique. Each envelope contained a paper slip allocating the sequence of the methods to be tested. For example: 25 envelopes contained the sequence: first $\mathrm{CM}$ and second ART, while other 25 envelopes indicated the sequence: first ART and second CM. 2) Sequence of the caries lesions: the supervisor randomly assigned the first tooth to either CM or ART using the flip of a coin.

\section{Clinical Procedures}

Cotton wool rolls were used for tooth isolation. Tooth surface was cleaned with a wet cotton pellet for removal of debris and plaque for both groups. In the test group (Chemomechanical - CM), cavity access was achieved using an enamel hatchet (Duflex-SS White, Petrópolis, RJ, Brazil). The exposed carious dentin was covered with Carisolv ${ }^{\mathrm{TM}}$ gel (MediTeam, Sävedalen, Sweden, batch \#1081465). After $30 \mathrm{~s}$, the carious dentin was gently scraped away using specially designed hand instruments (Multistar; MediTeam) to remove softened carious tissue. When the gel became heavily contaminated with debris, it was removed with 
cotton pellets and additional fresh gel was applied. The procedure was repeated until the gel no longer became cloudy and all surfaces of the cavity were hard on probing, indicating that there was no carious dentin left. In the control group (ART), cavity access was also achieved with the use of enamel hatchet. The next step was the removal of infected tissue with conventional spoon excavators (Duflex-SS White), first at the dentinoenamel junction and then from the floor of the cavity.

In both methods, after complete caries removal, the cavities were cleaned with small cotton pellets soaked in water and dried with dry cotton pellets (Cremer, Blumenau, $\mathrm{SC}$, Brazil). When necessary, pulpal protection with calcium hydroxide cement was used in deep cavities (Hydro C, Dentsply Ind. e Com. Ltda., Petrópolis, RJ, Brazil). Conditioning of the cavity and adjacent pits and fissures was carried out for $10 \mathrm{~s}$ with a cotton pellet saturated with the liquid component of the glass ionomer cement (Ketac Molar; 3M/ESPE, Seefeld, Germany). The conditioned surfaces were then washed with cotton pellets soaked in water and dried with dry cotton pellets. To restore proximal surfaces of anterior teeth, wedges (TDV Dental, São Paulo, SP, Brazil) and plastic strips (TDV Dental) were used. Metal matrix bands (T-band; TDV Dental) and wedges were used when placing Class II restorations. Ketac Molar glass ionomer cement (3M/ESPE) was mixed according to the manufacturer's instructions and placed into the cavity using the smooth side of a spoon excavator (Duflex-SS White). The operator's gloved finger was coated with Petroleum jelly (Sidepal, Guarulhos, SP, Brazil) and a slight pressure was applied on top of the entire occlusal surface for $30 \mathrm{~s}$. This "press-finger" technique was used to condense the material into the cavity and any adjacent pits and fissures, resulting in a sealant restoration. GIC excess was removed with a spoon excavator or carver instruments (Duflex-SS White). After initial hardening of the material, the occlusion was checked with articulating paper (AccuFilm II; Farmingdale, NYUSA) and, if necessary, adjusted with a carver. Two layers of varnish (Copalite; Cooley \& Cooley,
Houston, TX, USA) were applied over the restoration to prevent dehydration. Local anesthesia was used only when patients reported discomfort. The patient was instructed not to eat for at least $1 \mathrm{~h}$.

\section{Evaluation and Data Analysis}

Clinical evaluation was carried out at baseline and after 12 months by two independent calibrated examiners not involved in the treatment. Initially, visible debris and plaque were removed with an explorer (Duflex-SS White). The teeth were cleaned with a small cotton pellet soaked in water and dried with a dry cotton pellet. Clinical evaluation was performed using WHO periodontal probes, sharp sickleshaped explorers, plane front-surface mirrors and a light source. The criteria used to evaluate the ART restorations were those of a previous study and are given in Table $1^{13}$. The ball of the CPI probe $(\varnothing=0.5 \mathrm{~mm})$ was used to measure the size of any marginal defect and the amount of dental tissue removal.

Inter-examiner agreement was assessed with kappa statistics. Data were analyzed using 95\% confidence interval (IC95\%) on the binomial distribution. Fisher's exact test was used for comparisons between operators and groups. A difference was considered statistically significant if $\mathrm{p}<0.05$.

\section{RESULTS}

\section{Baseline}

A total of 50 pregnant women, with a mean age of $22 \pm$ 5.3 years (16-39 years) participated of this trial. The mean DMFT was $11.8 \pm 6.5$, with $6.7 \pm 3.9$ of the index related to decayed teeth.

In the test group (CM) 34 molars, 7 premolars and 9 incisors were treated, whereas in the control group (ART) 32 molars, 5 premolars and 13 incisors were treated. This difference was not significant statistically $\left(\mathrm{X}^{2}=1.12 ; \mathrm{P}=0.57\right)$.

No statistically significant differences were found between the test $(\mathrm{CM})$ and control (ART) groups regarding

TABLE 1- Evaluation criteria for ART restorations ${ }^{13}$

Score Description

0

1

2

3

4

5

6

7

8
Present, in good condition

Present, slight marginal defect, no repair is needed

Present, slight wear, no repair is needed

Present, marginal defect $>0.5 \mathrm{~mm}$, repair is needed

Present, wear $>0.05 \mathrm{~mm}$, repair is needed

Not present, restoration partly or completely missing

Not present, restoration replaced by another restoration

Tooth is extracted

Restoration not assessed, patient is nor present

Success: scores 0, 1 and 2; Failure: scores 3, 4, 5 and 6; Excluded: scores 7 and 8 
cavity class type $\left(\mathrm{X}^{2}=0.96 ; \mathrm{P}=0.62\right)$. In the test group $(\mathrm{CM})$, 36 Class I, 5 Class II and 9 Class III and class IV cavities were restored, while in the control group (ART) 32 Class I, 5 Class II and 13 incisors were restored. Both methods were compared in each individual, one immediately after the other, in 46 matched pairs of cavity class type and in 4 pairs of non-matched cavity class type.

The average time spent per restoration was $11.6 \pm 2.7$ $\min$ for the test group $(\mathrm{CM})$ and $10.2 \pm 3.1 \mathrm{~min}$ for the control group (ART). Comparing the mean times for caries removal, statistically significant difference was found with Student's t-test $(P<0.05)$.

Postoperative sensitivity was reported in the control group during the first day by 2 patients with deep carious lesions; in such cases local anesthetic was required and calcium hydroxide cement was also applied. No significant difference was found in the postoperative sensitivity between the groups (Fisher's exact test: $\mathrm{P}=0.24$ ). Ninety four percent of the patients were willing to receive both treatments again if required.

\section{Follow-up}

At 12-month follow-up, the success rates of the restorations were $100 \%(\mathrm{CI} 95 \%=93.3-100 \%)$ and $97.6 \%$ $(\mathrm{CI} 95 \%=87.4-99.9 \%)$ for test group (CM) and control group (ART), respectively. There was no statistically significant difference between groups (Fisher's exact test: $\mathrm{P}=0.49$ ), and operators (Fisher's exact test: $\mathrm{P}=1.00$ ). Results of the duplicate examinations on restoration status showed very good inter-examiner reproducibility with kappa values of 0.97 in the evaluation after 12 months. No caries lesion adjacent to the restoration was observed.

\section{DISCUSSION}

In this study, 86 out of the 100 restorations were evaluated at the 12-month follow-up. The lost-to-follow-up rate of $14 \%$ was low compared to that of previous ART studies that showed rates from $24.3 \%$ to $33.7 \%$ within the same evaluation period ${ }^{10,13,15,29}$. Substantial efforts were made to follow the study subjects by telephone calls and multiple site visits were made to reduce drop out. Seven patients (14 restorations) were excluded because they had left the city (score 8). In addition, one restoration was excluded from the control group (ART) because the tooth had been extracted (score 7). In this case, the patient reported that the extraction of the tooth occurred approximately 9 months after the treatment. This patient did not report pain or postoperative sensitivity. The other restoration placed in the mandibular arch was rated as a success.

The present study is the first that reports, in permanent teeth of adults, the influence of two minimally invasive cavity preparations (ART and CM) on the clinical performance of GIC restorations. Previous studies compared manual methods (ART or CM) with conventional rotary instruments, but did not compare the differences between the ART and $\mathrm{CM}$ in terms of survival of restorations.
After 12 months, the tested methods of caries removal (CM and ART) did not influence the success rates of the GIC restorations. The survival of the restorations was not statistically significant different, thus the null hypothesis was accepted. These findings are relevant because the high quality of the tooth/GIC interface provides tooth surfaces capable of developing durable adhesive bonds to $\mathrm{GIC}^{6}$. Additionally, in the present study, the survival of the restorations was not operator-dependent. These results suggest that operators who have received adequate training and calibration in minimally invasive cavity preparation and restoration methods can produce reliable results.

It is important to emphasize that, in the present study, all restorations were recorded as successful in test group (CM) and 1 out of 42 GIC restorations in control group (ART) was recorded as unsuccessful. In this case, the restoration recorded as failure presented marginal defect higher than $0.5 \mathrm{~mm}$. In such restoration, one occlusal site did not present enough bulk. This problem is likely to have contributed to the marginal defect of the restoration, but caries was not observed adjacent to the restoration. Our result is in accordance with those of previous ART studies ${ }^{10,13,14,29}$.

Despite the fact that infected dentin may have been left behind in the cavity, and that the patients in this study were considered of high-caries activity (mean DMFT score=11.86), secondary caries, whether residual or primary in origin, does not seem to have affected the survival of ART restorations after 12 months. These findings are in disagreement with the results of studies that still considered essential the removal of all carious dentin during restorative treatment ${ }^{27,28}$, and in agreement with clinical and microbiological studies that have shown clinical success and a significant reduction in bacteria after hand excavation in spite of bacterial remaining in incompletely excavated dentin ${ }^{16,17,24}$. Furthermore, it can be suggested that GICs present antibacterial activity ${ }^{7,22}$ and these materials are potentially able to remineralize residual carious dentin ${ }^{23}$.

Another important aspect to be considered in the success rate of GIC restorations in the present study was the presence of a chair-side assistant. This allowed the operator spending more time on saliva control after conditioning, while the assistant was mixing the GICs. Likewise, other authors have reported difficulty to control saliva or blood contamination in cavities with margins close to the cervical area, thereby having a detrimental effect on GIC bonding ${ }^{4,5,13,15,29}$. The use of minimally invasive cavity preparation techniques, such as ART and chemomechanical method, for providing restorative care to pregnant women should be encouraged. Both approaches were proved highly appropriate, effective and acceptable. It is reasonable to assume that the high acceptance by patients (94\%) may be attributed to the nonuse of rotary instruments or the local anesthetics. These factors may explain why the treatment was well accepted and are in accordance with previous studies s,5, $8,10,13,14,20,25^{\text {. }}$

Mickenautsch, et al. ${ }^{18}$ (2007) emphasized that the insufficient supply of dental materials and instruments, and dentists' perceptions of low levels of clinical skills in performing ART were barriers to use this treatment in public 
oral health services. This suggests that although the early 12-month findings are promising, further studies of longer duration are needed to confirm these outcomes, as well as long-term politics of oral health care will help improving the access of a grater part of the population to proper oral care.

\section{CONCLUSIONS}

From the data gathered from this clinical pilot study, it may be concluded that: 1 . Both minimally invasive cavity preparation methods, chemomechanical and ART, were proved highly appropriate, effective and acceptable for GIC restorations at the 12-month follow-up; 2 . The high survival of the GIC restorations was independent on the tested minimally invasive cavity preparation methods (chemomechanical or ART); 3. Further clinical trials should be conducted to validate the results of this pilot study.

\section{ACKNOWLEDGEMENTS}

The authors would like to thank the pregnant women who volunteered to take part in this work, and the staff of the Public Health Centers for participating in this project. TJE Barata was supported by a fellowship from Coordenação de Aperfeiçoamento de Pessoal de Nível Superior (CAPES), Brazil.

\section{REFERENCES}

1- Albuquerque OMR, Abegg CR, Rodrigues CS. Percepção de gestantes do Programa Saúde da Família em relação a barreiras no atendimento odontológico em Pernambuco, Brasil. Cad Saude Publica. 2004;20:789-96.

2- Banerjee A, Kidd EA, Watson TF. Scanning electron microscopic observations of human dentine after mechanical caries excavation. J Dent. 2000;28:179-86.

3- Beeley JA, Yip HK, Stevenson AG. Chemochemical caries removal: a review of the techniques and latest developments. Br Dent J. 2000;188:427-30.

4- Bresciani E, Carvalho WL, Pereira LCG, Barata TJE, GarcíaGodoy F, Navarro MFL. Six-month evaluation of ART one-surface restorations in a community with high caries experience in Brazil. J Appl Oral Sci. 2005;13:180-6.

5- Cefaly DFG, Barata TJE, Tapety CMC, Bresciani E, Navarro MFL. Clinical evaluation of multisurface ART restorations. J Appl Oral Sci. 2005;13:15-9.

6- Czarnecka B, Limanowska Shaw H, Nicholson JW. Microscopic evaluation of the interface between glass-ionomer cements and tooth structures prepared using conventional instruments and the atraumatic restorative treatment (ART) technique. Quintessence Int. 2006;37:557-64.

7- Duque C, Negrini TC, Hebling J, Spolidorio DM. Inhibitory activity of glass-ionomer cements on cariogenic bacteria. Oper Dent. 2005;30:636-40.
8- Ericson D, Zimmerman M, Raber H, Gotrick B, Bornstein R, Thorell J. Clinical evaluation of efficacy and safety of a new method for chemomechanical removal of caries: a multi-centre study. Caries Res. 1999;33:171-7.

9- Frencken JE, Holmgren CJ. The ART approach step-by-step. In: Frencken JE, Holmgren CJ. Atraumatic restorative treatment for dental caries. Nijmegen: STI Book; 1999. p. 39-54.

10- Frencken JE, Makoni F, Sithole WD. Atraumatic restorative treatment and glass-ionomer sealants in a school oral health programme in Zimbabwe: evaluation after 1 year. Caries Res. 1996;30:428-33.

11 - Fure S, Lingstrom P. Evaluation of the chemomechanical removal of dentine caries in vivo with a new modified Carisolv gel. Clin Oral Investig. 2004;8:139-44.

12- Fure S, Lingstrom P, Birkhed D. Evaluation of Carisolv for the chemomechanical removal of primary root caries in vivo. Caries Res. 2000;34:275-80.

13-Lo EC, Holmgren CJ. Provision of Atraumatic Restorative Treatment (ART) restorations to Chinese pre-school children a 30month evaluation. Int J Paediatr Dent. 2001;11:3-10.

14-Lo EC, Luo Y, Tan HP, Dyson JE, Corbet EF. ART and conventional root restorations in elders after 12 months. J Dent Res. 2006;85:929-32.

15- Mallow PK, Durward CS, Klaipo M. Restoration of permanent teeth in young rural children in Cambodia using the atraumatic restorative treatment (ART) technique and Fuji II glass ionomer cement. Int J Paediatr Dent. 1998;8:35-40.

16- Maltz M, de Oliveira EF, Fontanella V, Bianchi R. A clinical, microbiologic, and radiographic study of deep caries lesions after incomplete caries removal. Quintessence Int. 2002;33:151-9.

17- Massara ML, Alves JB, Brandão PR. Atraumatic restorative treatment: clinical, ultrastructural and chemical analysis. Caries Res. 2002;36:430-6.

18- Mickenautsch S, Frencken JE, Van't Hof M. Factors inhibiting the implementation of the Atraumatic Restorative Treatment approach in public oral health services in Gauteng province, South Africa. J Appl Oral Sci. 2007;15:1-8.

19- Mjör IA, Odont D. Pulp-dentin biology in restorative dentistry. Part 2: initial reactions to preparation of teeth for restorative procedures. Quintessence Int. 2001;32:537-51.

20 - Nadanovsky P, Cohen Carneiro F, Souza de Mello F. Removal of caries using only hand instruments: a comparison of mechanical and chemo-mechanical methods. Caries Res. 2001;35:384-9.

21- Oliveira BH, Nadanovsky P. The impact of oral pain on quality of life during pregnancy in low-income Brazilian women. J Orofac Pain. 2006;20:297-305.

22- Silva RC, Zuanon AC, Spolidorio DM, Campos JA. Antibacterial activity of four glass ionomer cements used in atraumatic restorative treatment. J Mater Sci Mater Med. 2007;18:1859-62.

23 - Smales RJ, Ngo HC, Yip KH, Yu C. Clinical effects of glass ionomer restorations on residual carious dentin in primary molars. Am J Dent. 2005;18:188-93.

24- Toi CS, Bönecker M, Cleaton-Jones PE. Mutans streptococci strains prevalence before and after cavity preparation during Atraumatic Restorative Treatment. Oral Microbiol Immunol. 2003;18:160-4. 
25- Topaloglu-Ak A, Eden E, Frencken JE. Perceived dental anxiety among schoolchildren treated through three caries removal approaches. J Appl Oral Sci. 2007;15:235-40.

26- Tyas MJ, Anusavice KJ, Frencken JE, Mount GJ. Minimal intervention dentistry-a review. FDI Commission Project 1-97. Int Dent J. 2000;50:1-12.

27-Weerheijm KL, Groen HJ. The residual caries dilemma. Community Dent Oral Epidemiol. 1999;27:436-41.

28 - Weerheijm KL, Kreulen CM, de Soet JJ, Groen HJ, van Amerongen WE. Bacterial counts in carious dentine under restorations: 2-year in vivo effects. Caries Res. 1999;33:130-4.

29- Yip KH, Smales RJ, Gao W, Peng D. The effects of two cavity preparation methods on the longevity of glass ionomer cement restorations: an evaluation after 12 months. J Am Dent Assoc. 2002;133:744-51. 